\title{
Nonparametric estimation of trend function for stochastic differential equations driven by a bifractional Brownian motion
}

\author{
Abdelmalik Keddi \\ Laboratory of Stochastic Models, \\ Statistic and Applications, \\ Dr. Moulay Tahar University of Saida \\ B. P. 138, En-Nasr, Saida 20000, Algeria \\ email: malokkeddi@yahoo.com
}

\section{Fethi Madani}

Laboratory of Stochastic Models,

Statistic and Applications,

Dr. Moulay Tahar University of Saida

B. P. 138, En-Nasr, Saida 20000, Algeria

email: fethi.madani@univ-saida.dz
Amina Angelika Bouchentouf

Laboratory of Mathematics, Djillali Liabes University of Sidi Bel Abbes, B. P. 89, Sidi Bel Abbes 22000, Algeria email: bouchentouf_amina@yahoo.fr

Abstract. The main objective of this paper is to investigate the problem of estimating the trend function $S_{t}=S\left(x_{t}\right)$ for process satisfying stochastic differential equations of the type

$$
d X_{t}=S\left(X_{t}\right) d t+\varepsilon d B_{t}^{H, K}, X_{0}=x_{0}, 0 \leq t \leq T,
$$

where $\left\{\mathrm{B}_{\mathrm{t}}^{\mathrm{H}, \mathrm{K}}, \mathrm{t} \geq 0\right\}$ is a bifractional Brownian motion with known parameters $H \in(0,1), K \in(0,1]$ and $H K \in(1 / 2,1)$. We estimate the unknown function $S\left(x_{t}\right)$ by a kernel estimator $\widehat{S}_{t}$ and obtain the asymptotic properties as $\varepsilon \longrightarrow 0$. Finally, a numerical example is provided.

2010 Mathematics Subject Classification: 62M09, 60G15

Key words and phrases: bifractional Brownian motion, trend function, kernel estimator, stochastic differential equations, nonparametric estimation 


\section{Introduction}

Fractional Brownian motion (fBm) is the most well-known and employed process with a long dependency-property for many real world applications including telecommunication, turbulence, finance, and so on. This process was introduced by Kolmogorov [5], then studied by many researchers including Mandelbrot and Van Ness [9] and Norros et al. [12].

The bifractional Brownian motion (bfBm) was introduced in Houdré and Villa [3], and further studied by Russo and Tudor [14] and Tudor and Xiao [16].

Nonparametric estimation of trend function for stochastic differential equations (SDEs) has caught the attention of different researchers. It was first investigated by Kutoyants [7] for the stochastic differential equation driven by a standard Brownian motion. After that, the problem was generalized by Mishra and Rao [10] for the stochastic differential equation driven by a fractional Brownian motion. Then, Mishra and Rao [11] presented nonparametric estimation of linear multiplier for fractional diffusion processes. Later, nonparametric inference for fractional diffusion were dealt by Saussereau [15]. Very recently, Prakasa Rao [13] investigated nonparametric estimation of trend function for SDEs driven by mixed fractional Brownian motion.

In this paper, we use the method developed by Kutoyants [7] to construct an estimate of the trend function $S_{t}$ in a model described by stochastic differential equations driven by a bifractional Brownian motion. For this, let $\left\{X_{t}, 0 \leq t \leq T\right\}$ be the process governed by the following equation:

$$
d X_{t}=S\left(X_{t}\right) d t+\varepsilon d B_{t}^{H, K}, X_{0}=x_{0}, 0 \leq t \leq T,
$$

where $\varepsilon>0$ and $\mathrm{B}_{\mathrm{t}}^{\mathrm{H}, \mathrm{K}}$ is a bifractional Brownian motion of parameters $\mathrm{H} \in$ $(0,1), K \in(0,1]$, and $S($.$) is an unknown function. In Kutoyants [7], the trend$ coefficient in a diffusion process was estimated from the process $\left\{X_{t}, 0 \leq t \leq T\right\}$. In this investigation, we use a similar approach and consider the estimate $\widehat{S}_{t}$ of $S_{t}$ as follows:

$$
\widehat{S}_{t}=\frac{1}{\phi_{\varepsilon}} \int_{0}^{T} G\left(\frac{\tau-t}{\phi_{\varepsilon}}\right) d X_{\tau},
$$

where $G$ is a bounded kernel with finite support with $\phi_{\varepsilon} \longrightarrow 0$ as $\varepsilon \longrightarrow 0$. Under some hypotheses, we firstly prove the mean square consistency of the estimator. Then, we give a bound on the rate of convergence and prove the asymptotic normality of the estimator $\hat{S}_{t}$.

To the best of our knowledge, the problem of nonparametric estimation of 
trend function for stochastic differential equations driven by a bfBm has not been considered in the literature.

The rest of the paper is structured as follows. In Section 2, the basic properties of bifractional Brownian motion are stated. Section 3 is devoted to the preliminaries. Then, in Section 4, we give the main results; under some hypotheses, we establish the uniform consistency (Theorem 1), the rate of convergence (Theorem 2) as well as the asymptotic normality (Theorem 3) of the estimator. Further, in Section 5, a simulation example is carried out to illuminate our theoretical study. Section 6 is devoted to the technical proofs. Finally, we conclude the paper in Section 7.

\section{Bifractional Brownian motion}

Let $\left(\Omega, \mathcal{F},\left\{\mathcal{F}_{t}\right\}_{t} \geq 0, \mathbb{P}\right)$ be a stochastic basis satisfying the habitual hypotheses, i.e., a filtered probability space with a right continuous filtration $\left\{\mathcal{F}_{t}\right\}_{t} \geq 0$ and $\mathcal{F}_{0}$ contains every $\mathbb{P}$-null set.

Let $\left\{B_{t}^{H, K}, t \geq 0\right\}$ be a normalized bifractional Brownian motion with parameters $H \in(0,1)$ and $K \in(0,1]$, that is, a Gaussian process with continuous sample paths with $\mathrm{B}_{0}^{\mathrm{H}, \mathrm{K}}=0$ and the covariance:

$$
\mathrm{R}_{\mathrm{H}, \mathrm{K}}(\mathrm{t}, \mathrm{s})=\mathbb{E}\left(\mathrm{B}_{\mathrm{t}}^{\mathrm{H}, \mathrm{K}} \mathrm{B}_{s}^{\mathrm{H}, \mathrm{K}}\right)=\frac{1}{2^{\mathrm{K}}}\left[\left(\mathrm{t}^{2 \mathrm{H}}+\mathrm{s}^{2 \mathrm{H}}\right)^{\mathrm{K}}+|\mathrm{s}-\mathrm{t}|^{2 \mathrm{HK}}\right], \quad \mathrm{t} \geq 0, \quad s \geq 0 .
$$

When $K=1$, we retrieve the fractional Brownian motion while the case $\mathrm{K}=1$ and $\mathrm{H}=1 / 2$ corresponds to the standard Brownian motion.

The bfBm is an extension of the $\mathrm{fBm}$ which preserves many properties of the $\mathrm{fBm}$, but not the stationarity of the increments. Russo and Tudor [14] showed that the $\mathrm{bfBm} \mathrm{B}^{\mathrm{H}, \mathrm{K}}$ behaves as a $\mathrm{fBm}$ of Hurst parameter HK.

According to Houdré and Villa [3] and Tudor and Xiao [16], the bfBm has the following properties:

1. $\mathbb{E}\left(B_{t}^{\mathrm{H}, \mathrm{H}}\right)=0$ and $\operatorname{Var}\left(\mathrm{B}_{\mathrm{t}}^{\mathrm{H}, \mathrm{K}}\right)=\mathrm{t}^{2 \mathrm{HK}}$.

2. $B_{t}^{H, K}$ is said to be self-similar with index $H K \in(0,1)$, that is, for every constant $a>0$,

$$
\left\{B_{a t}^{H, K}, t \geq 0\right\} \triangleq\left\{a^{H K} B_{t}^{H, K}, t \geq 0\right\} \text {, for each } a>0,
$$

in the sense that the processes, on both sides of the equality sign, have the same finite dimensional distributions. 
3. The process $B_{t}^{H, K}$ is not Markov and it is not a semi-martingale if $\mathrm{HK} \neq$ $1 / 2$.

4. The trajectories of the process $\mathrm{B}^{\mathrm{H}, \mathrm{K}}$ are Hölder continuous of order $\delta$ for any $\delta<$ HK and they are nowhere differentiable.

5. The $\mathrm{bfBm} \mathrm{B}^{\mathrm{H}, \mathrm{K}}$ is a quasi-helix in the sense of Kahane [4], for any $t, s \geq 0$ we have

$$
2^{-K}(t-s)^{2 H K} \leq \mathbb{E}\left[B_{t}^{H, K}-B_{s}^{H, K}\right]^{2} \leq 2^{1-K}(t-s)^{2 H K} .
$$

The bfBm $\mathrm{B}^{\mathrm{H}, \mathrm{K}}$ can be extended for $\mathrm{K} \in(1,2)$ with $\mathrm{H} \in(0,1)$ and $\mathrm{HK} \in(0,1)$ (see Bardina and Es-Sebaiy [1] and Lifshits and Volkova [8]).

The stochastic calculus with respect to the bifractional Brownian motion has been recently developed by Kruk et al. [6]. More works on bifractional Brownian motion can be found in Tudor and Xiao [16], Es-sabaiy and Tudor [2], Yan et al. [17] and the references therein.

Fix a time interval $[0, T]$, we denote by $\mathcal{E}$ the set of step function on $[0, T]$. Let $\mathcal{H}_{\mathrm{B} H, \mathrm{~K}}$ be the canonical Hilbert space associated to the bfBm defined as the closure of $\mathcal{E}$ with respect to the scalar product

$$
\left\langle 1_{[0, \mathrm{t}]}, 1_{[0, \mathrm{~s}]}\right\rangle_{\mathcal{H}_{\mathrm{B} H, \mathrm{~K}}}=\mathrm{R}_{\mathrm{H}, \mathrm{K}}(\mathrm{t}, \mathrm{s})=\int_{0}^{T} \int_{0}^{T} 1_{[0, \mathrm{t}]}(\mathrm{u}) 1_{[0, \mathrm{~s}]}(v) \frac{\partial^{2} \mathrm{R}_{\mathrm{H}, \mathrm{K}}(\mathrm{u}, v)}{\partial u \partial v} \mathrm{dud} v,
$$

where $R_{H, K}(t, s)$ is the covariance of $B_{t}^{H, K}$ and $B_{s}^{H, K}$. The application $\varphi \in \mathcal{E} \longrightarrow$ $\mathrm{B}^{\mathrm{H}, \mathrm{K}}(\varphi)$ is an isometry from $\mathcal{E}$ to the Gaussian space generated by $\mathrm{B}^{\mathrm{H}, \mathrm{K}}$ and it can be extended to $\mathcal{H}_{\mathrm{B}, \mathrm{K}}$. In this study, as $\mathrm{HK} \in(1 / 2,1)$ we will employ the subspace $\left|\mathcal{H}_{\mathrm{B}, \mathrm{K}}\right|$ of $\mathcal{H}_{\mathrm{B}, \mathrm{K}, \mathrm{K}}$ which is defined as the set of measurable function $\varphi$ on $[0, T]$ satisfying

$$
\|\varphi\|_{\left|\mathcal{H}_{\mathrm{B} H, \mathrm{~K}}\right|}:=\int_{0}^{T} \int_{0}^{T}|\varphi(u)||\varphi(v)| \frac{\partial^{2} \mathrm{R}_{\mathrm{H}, \mathrm{K}}(u, v)}{\partial u \partial v} \mathrm{dud} v<\infty,
$$

such that

$$
\frac{\partial^{2} R_{H, K}(u, v)}{\partial u \partial v}=\alpha_{H, K}\left(t^{2 H}+s^{2 H}\right)^{K-2}(t s)^{2 H-1}+\beta_{H, K}|t-s|^{2 H K-2},
$$

where

$$
\alpha_{\mathrm{H}, \mathrm{K}}=2^{-\mathrm{K}+2} \mathrm{H}^{2} \mathrm{~K}(\mathrm{~K}-1) \quad \text { and } \quad \beta_{\mathrm{H}, \mathrm{K}}=2^{-\mathrm{K}+1} \mathrm{HK}(2 \mathrm{HK}-1) .
$$


Note that, if $\varphi, \psi \in\left|\mathcal{H}_{\mathrm{B} H, \mathrm{~K}}\right|$, then their scalar product in $\mathcal{H}_{\mathrm{B} H, \mathrm{~K}}$ is given by

$$
\langle\varphi, \psi\rangle_{\mathcal{H}_{\mathrm{B} H, \mathrm{~K}}}=\int_{0}^{T} \int_{0}^{T} \varphi(u) \psi(v) \frac{\partial^{2} R_{\mathrm{H}, \mathrm{K}}(u, v)}{\partial u \partial v} \mathrm{dud} v .
$$

For $\varphi, \psi \in\left|\mathcal{H}_{\mathrm{B}} \mathrm{H}, \mathrm{K}\right|$, we have

$$
\mathbb{E}\left(\int_{0}^{T} \varphi(u) \mathrm{dB}_{\mathfrak{u}}^{\mathrm{H}, \mathrm{K}}\right)=0, \mathbb{E}\left(\int_{0}^{T} \varphi(u) \mathrm{dB}_{\mathfrak{u}}^{\mathrm{H}, \mathrm{K}} \int_{0}^{T} \psi(v) \mathrm{dB}_{v}^{\mathrm{H}, \mathrm{K}}\right)=\langle\varphi, \psi\rangle_{\mathcal{H}_{\mathrm{B}, \mathrm{K}}} \cdot
$$

It is worth being pointed out that the canonical Hilbert space $\mathcal{H}_{\mathrm{B}, \mathrm{K}}$ associated with $\mathrm{B}^{\mathrm{H}, \mathrm{K}}$ satisfies:

$$
\mathrm{L}^{2}([0, \mathrm{~T}]) \subset \mathrm{L}^{1 / \mathrm{HK}}([0, \mathrm{~T}]) \subset\left|\mathcal{H}_{\mathrm{B}, \mathrm{K}}\right| \subset \mathcal{H}_{\mathrm{B} H, \mathrm{~K}},
$$

where $H \in(0,1), K \in(0,1]$ and $H K \in(1 / 2,1)$.

\section{Preliminaries}

Let $\left\{X_{t}, 0 \leq t \leq T\right\}$ be a process governed by the following equation:

$$
d X_{t}=S\left(X_{t}\right) d t+\varepsilon d B_{t}^{H, K}, \quad X_{0}=x_{0}, 0 \leq t \leq T,
$$

where $\varepsilon>0, B_{t}^{H, K}$ a bifractional Brownian motion, and $S($.$) is an unknown$ function. We suppose that $x_{t}$ is a solution of the following equation

$$
\frac{\mathrm{d} x_{\mathrm{t}}}{\mathrm{dt}}=\mathrm{S}\left(\mathrm{x}_{\mathrm{t}}\right), \mathrm{x}_{0}, 0 \leq \mathrm{t} \leq \mathrm{T} .
$$

We also suppose that the function $S: \mathbb{R} \longrightarrow \mathbb{R}$ satisfies the following assumptions:

(A1) There exists $L>0$ such that

$$
|S(x)-S(y)| \leq L|x-y|, \quad x, y \in \mathbb{R}
$$

(A2) There exists $M>0$ such that

$$
|S(x)| \leq M(1+|x|), x \in \mathbb{R},
$$

Then, the stochastic differential equation (4) has a unique solution $\left\{X_{t}, 0 \leq t \leq T\right\}$.

(A3) Assume that the function $S(x)$ is bounded by a constant $C$. 
Since the function $x_{t}$ satisfies (5), it follows that

$$
\left|S\left(x_{t}\right)-S\left(x_{s}\right)\right| \leq L\left|x_{t}-x_{s}\right|=L\left|\int_{S}^{t} S\left(x_{r}\right) d r\right| \leq L C|t-s|, t, s \in[0, T] .
$$

Let us define $\Sigma_{0}(\mathrm{~L})$ as the class of all functions $S(x)$ satisfying the assumption (A1) and uniformly bounded by the same constant C. Further, we denote by $\Sigma_{k}(\mathrm{~L})$ the class of all function $\mathrm{S}(\mathrm{x})$ which are uniformly bounded by the same constant $\mathrm{C}$ and which are $\mathrm{k}$-times differentiable with respect to $\mathrm{x}$ satisfying the following condition

$$
\left|S^{k}(x)-S^{k}(y)\right| \leq L|x-y|, x, y \in \mathbb{R},
$$

where $S^{k}(x)$ is the k-th derivative of $S(x)$.

Lemma 1 Assume that hypothesis (A1) is verified. Let $\mathrm{X}_{\mathrm{t}}$ and $\mathrm{x}_{\mathrm{t}}$ be the solutions of the equations (4) and (5) respectively. Then, we have

$$
\sup _{0 \leq t \leq T} \mathbb{E}\left(X_{t}-X_{t}\right)^{2} \leq e^{2 L T} \varepsilon^{2} T^{2 H K} .
$$

\section{Proof of the Lemma 1}

By (4) and (5), we have

$$
X_{t}=x_{0}+\int_{0}^{t} S\left(X_{r}\right) d r+\varepsilon B_{t}^{H, K}
$$

and

$$
x_{t}=x_{0}+\int_{0}^{t} S\left(x_{r}\right) d r
$$

This implies

$$
X_{t}-x_{t}=\int_{0}^{t}\left(S\left(X_{r}\right)-S\left(x_{r}\right)\right) d r+\varepsilon B_{t}^{H, K}
$$

Thus

$$
\begin{aligned}
\left|X_{t}-x_{t}\right| & \leq \int_{0}^{t}\left|S\left(X_{r}\right)-S\left(x_{r}\right)\right| d r+\varepsilon\left|B_{t}^{H,}, K^{t}\right| \\
& \leq L \int_{0}^{t}\left|X_{r}-x_{r}\right| d r+\varepsilon\left|B_{t}^{H, K}\right| .
\end{aligned}
$$

Putting $\mathfrak{u}_{t}=\left|X_{t}-x_{t}\right|$, we have

$$
u_{t} \leq \int_{0}^{t} u_{r} d r+\varepsilon\left|B_{t}^{H, K}\right|
$$


By using Grönwall's inequality, we obtain

$$
\left|X_{t}-x_{t}\right| \leq e^{L t} \varepsilon\left|B_{t}^{H, K}\right| .
$$

Then, since $\mathbb{E}\left(B_{t}^{H, K}\right)^{2}=t^{2 H K}$, we have

$$
\mathbb{E}\left|X_{t}-\chi_{t}\right|^{2} \leq e^{2 L t} \varepsilon^{2} t^{2 H K} .
$$

Finally, we find

$$
\sup _{0 \leq t \leq T} \mathbb{E}\left(X_{t}-x_{t}\right)^{2}<e^{2 L T} \varepsilon^{2} T^{2 H K}
$$

\section{Main results}

The main goal of this work is to build an estimator of the trend function $S_{t}$ in the model described by stochastic differential equation (4) using the method developed by Kutoyants [7]. Then, we study its asymptotic properties as $\varepsilon \longrightarrow 0$.

For all $t \in[0, T]$, the kernel estimator $\hat{S}_{t}$ of $S_{t}$ is given by

$$
\widehat{S}_{t}=\frac{1}{\phi_{\varepsilon}} \int_{0}^{T} G\left(\frac{\tau-t}{\phi_{\varepsilon}}\right) d X_{\tau},
$$

where $G(u)$ is a bounded function with finite support $[A, B]$ satisfying the following hypotheses:

(H1) $G(u)=0$ for $u<A$ and $u>B$ and $\int_{A}^{B} G(u) d u=1$,

(H2) $\int_{-\infty}^{+\infty} \mathrm{G}^{2}(\mathrm{u}) \mathrm{d} u<\infty$,

(H3) $\int_{-\infty}^{+\infty} u^{2(k+1)} G^{2}(u) d u<\infty$,

(H4) $\int_{-\infty}^{+\infty}|\mathrm{G}(\mathrm{u})|^{\frac{1}{H K}} \mathrm{~d} u<\infty$,

Further, we suppose that the normalizing function $\phi_{\varepsilon}$ satisfies:

(H5) $\phi_{\varepsilon} \longrightarrow 0$ and $\varepsilon^{2} \phi_{\varepsilon}^{-1} \longrightarrow 0$ as $\varepsilon \longrightarrow 0$.

The following theorem gives the uniform convergence of the estimator $\hat{S}_{t}$.

Theorem 1 Suppose that the assumptions (A1)-(A3) and (H1)-(H5) hold true. Further, suppose that the trend function $\mathrm{S}(\mathrm{x})$ belongs to $\Sigma_{0}(\mathrm{~L})$. Then, for any 
$0<\mathrm{c} \leq \mathrm{d}<\mathrm{T}$ and $\mathrm{HK} \in(1 / 2,1)$, the estimator $\hat{\mathrm{S}}_{\mathrm{t}}$ is uniformly consistent, that is,

$$
\lim _{\varepsilon \rightarrow 0} \sup _{S(x) \in \Sigma_{0}(L)} \sup _{c \leq t \leq d} \mathbb{E}_{S}\left(\left|\hat{S}_{t}-S\left(x_{t}\right)\right|^{2}\right)=0 .
$$

The following additional assumptions are useful for the rest of the theoretical study. Assume that

(H6) $\int_{-\infty}^{+\infty} u^{j} G(u) d u=0$ for $j=1,2, \ldots, k$,
(H7) $\int_{-\infty}^{+\infty} u^{k+1} G(u) d u<\infty$ and $\int_{-\infty}^{+\infty} u^{2(k+2)} G^{2}(u) d u<\infty$.

The rate of convergence of the estimator $\widehat{S}_{t}$ is established in the following theorem.

Theorem 2 Suppose that the function $\mathrm{S}(\mathrm{x}) \in \Sigma_{\mathrm{k}}(\mathrm{L}), \mathrm{HK} \in(1 / 2,1)$ and $\phi_{\varepsilon}=$ $\varepsilon^{\frac{1}{\mathrm{k}-\mathrm{HK}+2}}$. Then, under the hypotheses $(\mathrm{A} 1)-(\mathrm{A} 3)$ and $(\mathrm{H} 1)-(\mathrm{H} 7)$, we have

$$
\limsup _{\varepsilon \longrightarrow 0} \sup _{S(x) \in \Sigma_{k}(L)} \sup _{c \leq t \leq d} \mathbb{E}_{S}\left(\left|\hat{S}_{t}-S\left(x_{t}\right)\right|^{2}\right) \varepsilon^{\frac{-2(k+1)}{k-H K+2}}<\infty \text {. }
$$

Finally, the following theorem presents the asymptotic normality of the kernel type estimator $\hat{S}_{t}$ of $S\left(x_{t}\right)$.

Theorem 3 Suppose that the function $\mathrm{S}(\mathrm{x}) \in \Sigma_{\mathrm{k+1}}(\mathrm{L}), \mathrm{HK} \in(1 / 2,1)$ and $\phi_{\varepsilon}=\varepsilon^{\frac{1}{k-\mathrm{HK}+2}}$. Then, under the hypotheses (A1)-(A3) and $(\mathrm{H} 1)-(\mathrm{H} 7)$, we have

$$
\varepsilon^{\frac{-(\mathrm{k}+1)}{\mathrm{k}-\mathrm{HK}+2}}\left(\hat{S}_{\mathrm{t}}-\mathrm{S}\left(\mathrm{x}_{\mathrm{t}}\right)\right) \stackrel{\mathcal{D}}{\longrightarrow} \mathcal{N}\left(\mathrm{m}, \sigma_{\mathrm{H}, \mathrm{K}}^{2}\right) \text {, as } \varepsilon \longrightarrow 0 \text {, }
$$

where

$$
m=\frac{S^{k+1}\left(x_{t}\right)}{(k+1) !} \int_{-\infty}^{+\infty} G(u) u^{k+1} d u
$$

and

$$
\begin{aligned}
\sigma_{\mathrm{H}, \mathrm{K}}^{2}= & \int_{-\infty}^{+\infty} \int_{-\infty}^{+\infty} \mathrm{G}(\mathrm{u}) \mathrm{G}(v)\left[\alpha_{\mathrm{H}, \mathrm{K}}\left(\mathrm{u}^{2 \mathrm{H}}+v^{2 \mathrm{H}}\right)^{\mathrm{K}-2}(\mathrm{u} v)^{2 \mathrm{H}-1}\right. \\
& \left.+\beta_{\mathrm{H}, \mathrm{K}}|\mathrm{u}-v|^{2 \mathrm{HK}-2}\right] \mathrm{d} u \mathrm{~d} v,
\end{aligned}
$$

with

$$
\alpha_{\mathrm{H}, \mathrm{K}}=2^{-\mathrm{K}+2} \mathrm{H}^{2} \mathrm{~K}(\mathrm{~K}-1) \quad \text { and } \quad \beta_{\mathrm{H}, \mathrm{K}}=2^{-\mathrm{K}+1} \mathrm{HK}(2 \mathrm{HK}-1) .
$$




\section{$5 \quad$ Numerical example}

The main objective of this part is to conduct a numerical study to illustrate our theoretical result. We compare our kernel estimator for stochastic differential equations driven by a bifractional Brownian motion to the kernel estimator for stochastic differential equations driven by fractional Brownian motion given in Mishra and Prakasa Rao [10]. We compare numerically the variance $\sigma_{\mathrm{H}, \mathrm{K}}^{2}$ of our estimator to $\sigma_{\mathrm{H}}^{2}$.

Consider a function $\mathrm{G}$ which satisfies hypotheses (H1)-(H7):

$$
G(t)=\frac{15}{128}\left(63 t^{4}+70 t^{2}+15\right),|t| \leq 1 .
$$

- The variance of the kernel estimator for stochastic differential equations driven by fractional Brownian motion given in Mishra and Prakasa Rao [10] is given as:

For all $H \in(1 / 2,1)$,

$$
\sigma_{H}^{2}=H(2 H-1) \int_{-\infty}^{+\infty} \int_{-\infty}^{+\infty} G(u) G(v)|u-v|^{2 H-2} d u d v,
$$

- Using the result given in Theorem 3, the variance of our estimator is obtained as:

For all $H \in(0,1), K \in(0,1]$ and $H K \in(1 / 2,1)$, we have

$$
\begin{aligned}
\sigma_{\mathrm{H}, \mathrm{K}}^{2}= & \int_{-\infty}^{+\infty} \int_{-\infty}^{+\infty} \mathrm{G}(\mathrm{u}) \mathrm{G}(v)\left[\alpha_{\mathrm{H}, \mathrm{K}}\left(\mathrm{u}^{2 \mathrm{H}}+v^{2 \mathrm{H}}\right)^{\mathrm{K}-2}(\mathrm{u} v)^{2 \mathrm{H}-1}\right. \\
& \left.+\beta_{\mathrm{H}, \mathrm{K}}|\mathrm{u}-v|^{2 \mathrm{HK}-2}\right] \mathrm{d} u \mathrm{~d} v,
\end{aligned}
$$

where

$$
\alpha_{\mathrm{H}, \mathrm{K}}=2^{-\mathrm{K}+2} \mathrm{H}^{2} \mathrm{~K}(\mathrm{~K}-1) \quad \text { and } \quad \beta_{\mathrm{H}, \mathrm{K}}=2^{-\mathrm{K}+1} \mathrm{HK}(2 \mathrm{HK}-1) .
$$

Next, we compute the variances, the results are presented in the following Tables 
Table 1: The variance values $\sigma_{\mathrm{H}}^{2}$.

\begin{tabular}{ccccccc}
\hline $\mathrm{H}$ & 0.7 & 0.75 & 0.8 & 0.85 & 0.9 & 0.95 \\
\hline$\sigma_{\mathrm{H}}^{2}$ & 1.1567 & 1.1900 & 1.1830 & 1.1506 & 1.1025 & 1.0452 \\
\hline
\end{tabular}

Table 2: The variance values $\sigma_{\mathrm{H}, \mathrm{K}}^{2}$.

\begin{tabular}{ccccccc}
\hline $\mathrm{K} \backslash \mathrm{H}$ & 0.7 & 0.75 & 0.8 & 0.85 & 0.9 & 0.95 \\
\hline 0.75 & 0.6006 & 0.9458 & 1.1647 & 1.2965 & 1.3709 & 1.4091 \\
\hline 0.8 & 0.8733 & 1.1230 & 1.2696 & 1.3462 & 1.3774 & 1.3801 \\
\hline 0.85 & 1.0362 & 1.2107 & 1.3019 & 1.3376 & 1.3378 & 1.3159 \\
\hline 0.9 & 1.1227 & 1.2382 & 1.2873 & 1.2930 & 1.2712 & 1.2326 \\
\hline 0.95 & 1.1570 & 1.2264 & 1.2437 & 1.2274 & 1.1901 & 1.1402 \\
\hline 1 & 1.1567 & 1.1900 & 1.1830 & 1.1506 & 1.1025 & 1.0452 \\
\hline
\end{tabular}

From the obtained results in Tables 1 and 2, we clearly see that the variance of our estimator is less than that of the kernel estimator for stochastic differential equations driven by fractional Brownian motion. We can conclude that our kernel estimator for stochastic differential equations driven by a bifractional Brownian motion is better than that given in Mishra and Prakasa Rao [10].

\section{Proof of Theorems}

\subsection{Proof of Theorem 1}

From (4) and (10), we can see that

$$
\begin{aligned}
\hat{S}_{\mathrm{t}}-S\left(x_{\mathrm{t}}\right)= & \frac{1}{\phi_{\varepsilon}} \int_{0}^{T} G\left(\frac{\tau-\mathrm{t}}{\phi_{\varepsilon}}\right) d X_{\tau}-S\left(x_{\mathrm{t}}\right) \\
= & \frac{1}{\phi_{\varepsilon}} \int_{0}^{T} G\left(\frac{\tau-\mathrm{t}}{\phi_{\varepsilon}}\right)\left(S\left(X_{\tau}\right) d \tau+\varepsilon d B_{\tau}^{\mathrm{H}, \mathrm{K}}\right)-S\left(x_{\mathrm{t}}\right) \\
= & \frac{1}{\phi_{\varepsilon}} \int_{0}^{T} G\left(\frac{\tau-\mathrm{t}}{\phi_{\varepsilon}}\right)\left(S\left(X_{\tau}\right)-S\left(x_{\tau}\right)\right) d \tau \\
& +\frac{1}{\phi_{\varepsilon}} \int_{0}^{T} G\left(\frac{\tau-t}{\phi_{\varepsilon}}\right) S\left(x_{\tau}\right) d \tau-S\left(x_{t}\right) \\
& +\frac{\varepsilon}{\phi_{\varepsilon}} \int_{0}^{T} G\left(\frac{\tau-t}{\phi_{\varepsilon}}\right) \mathrm{dB}_{\tau}^{\mathrm{H}, \mathrm{K}} .
\end{aligned}
$$


Using the inequality $(\alpha+\beta+\gamma)^{2} \leq 3 \alpha^{2}+3 \beta^{2}+3 \gamma^{2}$, it yields

$$
\begin{aligned}
\mathbb{E}_{S}\left[\hat{S}_{t}-S\left(x_{t}\right)\right]^{2} \leq 3 \mathbb{E}_{S} & {\left[\frac{1}{\phi_{\varepsilon}} \int_{0}^{T} G\left(\frac{\tau-t}{\phi_{\varepsilon}}\right)\left(S\left(X_{\tau}\right)-S\left(x_{\tau}\right)\right) d \tau\right]^{2} } \\
& +3 \mathbb{E}_{S}\left[\frac{1}{\phi_{\varepsilon}} \int_{0}^{T} G\left(\frac{\tau-t}{\phi_{\varepsilon}}\right) S\left(x_{\tau}\right) d \tau-S\left(x_{t}\right)\right]^{2} \\
& +3 \mathbb{E}_{S}\left[\frac{\varepsilon}{\phi_{\varepsilon}} \int_{0}^{T} G\left(\frac{\tau-t}{\phi_{\varepsilon}}\right) d B_{\tau}^{H, K}\right]^{2} \\
\leq I_{1} & +I_{2}+I_{3} .
\end{aligned}
$$

- Concerning $\mathrm{I}_{1}$. Via inequalities (6) and (8) and hypotheses (H1)-(H2), we get

$$
\begin{aligned}
\mathrm{I}_{1} & =3 \mathbb{E}_{S}\left[\frac{1}{\phi_{\varepsilon}} \int_{0}^{T} G\left(\frac{\tau-t}{\phi_{\varepsilon}}\right)\left(S\left(X_{\tau}\right)-S\left(x_{\tau}\right)\right) d \tau\right]^{2} \\
& =3 \mathbb{E}_{S}\left[\int_{-\infty}^{+\infty} G(u)\left(S\left(X_{t+\phi_{\varepsilon} u}\right)-S\left(x_{t+\phi_{\varepsilon} u}\right)\right) d u\right]^{2} \\
& \leq 3(B-A) \mathbb{E}_{S}\left[\int_{-\infty}^{+\infty} G^{2}(u)\left(S\left(X_{t+\phi_{\varepsilon} u}\right)-S\left(x_{t+\phi_{\varepsilon} u}\right)\right)^{2} d u\right] \\
& \leq 3(B-A) L^{2} \mathbb{E}_{S}\left[\int_{-\infty}^{+\infty} G^{2}(u)\left(X_{t+\phi_{\varepsilon} u}-x_{t+\phi_{\varepsilon} u}\right)^{2} d u\right] \\
& \leq 3(B-A) L^{2}\left[\int_{-\infty}^{+\infty} G^{2}(u) \sup _{0 \leq t+\phi_{\varepsilon} u \leq T} \mathbb{E}_{S}\left(X_{t+\phi_{\varepsilon} u}-x_{t+\phi_{\varepsilon} u}\right)^{2} d u\right] \\
& \leq 3(B-A) L^{2} e^{2 L T} T^{2 H K} \varepsilon^{2} \\
& \leq C_{1} \varepsilon^{2},
\end{aligned}
$$

where $C_{1}$ is a positive constant depending on $T, L, H, K$, and $(B-A)$.

- Concerning $\mathrm{I}_{2}$. Let

$$
\begin{aligned}
I_{2} & =3 \mathbb{E}_{S}\left[\frac{1}{\phi_{\varepsilon}} \int_{0}^{T} G\left(\frac{\tau-t}{\phi_{\varepsilon}}\right) S\left(x_{\tau}\right) d \tau-S\left(x_{t}\right)\right]^{2} \\
& =3 \mathbb{E}_{S}\left[\int_{-\infty}^{+\infty} G(u) S\left(x_{t+\phi_{\varepsilon} u}\right) d u-S\left(x_{t}\right)\right]^{2} \\
& =3 \mathbb{E}_{S}\left[\int_{-\infty}^{+\infty} G(u)\left(S\left(x_{t+\phi_{\varepsilon} u}\right)-S\left(x_{t}\right)\right) d u\right]^{2}
\end{aligned}
$$


Next, by using hypotheses (A3) and (H3), we have

$$
\begin{aligned}
\mathrm{I}_{2} & \leq 3 \mathrm{~L}^{2} \mathrm{C}_{2}^{2} \mathbb{E}_{\mathrm{S}}\left[\int_{-\infty}^{+\infty} \mathrm{G}(\mathrm{u})\left(\phi_{\varepsilon} \mathrm{u}\right) \mathrm{du}\right]^{2} \\
& \leq 3(\mathrm{~B}-\mathrm{A}) \mathrm{L}^{2} \mathrm{C}_{2}^{2}\left[\int_{-\infty}^{+\infty} \mathrm{G}^{2}(\mathrm{u}) \mathrm{u}^{2} \mathrm{du}\right] \phi_{\varepsilon}^{2} \\
& \leq \mathrm{C}_{3} \phi_{\varepsilon}^{2},
\end{aligned}
$$

where $C_{3}$ is a positive constant depending on $L$ and $(B-A)$.

- Concerning $\mathrm{I}_{3}$. Since $\mathrm{HK} \in(1 / 2,1)$, we have

$$
\begin{aligned}
\mathrm{I}_{3} & =3 \mathbb{E}_{\mathrm{S}}\left[\frac{\varepsilon}{\phi_{\varepsilon}} \int_{0}^{T} \mathrm{G}\left(\frac{\tau-\mathrm{t}}{\phi_{\varepsilon}}\right) \mathrm{dB}_{\tau}^{\mathrm{H}, \mathrm{K}}\right]^{2} \\
& =3 \frac{\varepsilon^{2}}{\phi_{\varepsilon}^{2}} \mathbb{E}_{S}\left[\int_{0}^{T} \mathrm{G}\left(\frac{\tau-\mathrm{t}}{\phi_{\varepsilon}}\right) \mathrm{dB}_{\tau}^{\mathrm{H}, \mathrm{K}}\right]^{2} \\
& \leq 3 \frac{\varepsilon^{2}}{\phi_{\varepsilon}^{2}}\left[\mathrm{C}(2, \mathrm{HK})\left(\int_{0}^{T}\left|\mathrm{G}\left(\frac{\tau-\mathrm{t}}{\phi_{\varepsilon}}\right)\right|^{\frac{1}{\mathrm{HK}}} \mathrm{d} \tau\right)^{2 \mathrm{HK}}\right] \\
& \leq \mathrm{C}_{4} \frac{\varepsilon^{2}}{\phi_{\varepsilon}^{2}}\left[\phi_{\varepsilon}^{2 \mathrm{HK}}\left(\int_{-\infty}^{+\infty}|\mathrm{G}(\mathrm{u})|^{\frac{1}{\mathrm{HK}}} \mathrm{du}\right)^{2 \mathrm{HK}}\right] \\
& \leq \mathrm{C}_{5} \frac{\varepsilon^{2}}{\phi_{\varepsilon}} \phi_{\varepsilon}^{2 \mathrm{HK}-1} \text { (using hypothesis (H4)), }
\end{aligned}
$$

where $\mathrm{C}_{5}$ is a positive constant depending on $\mathrm{H}$ and $\mathrm{K}$.

Combining (13)-(16), we have

$$
\sup _{S(x) \in \Sigma_{0}(\mathrm{~L})} \sup _{\mathrm{c} \leq \mathrm{t} \leq \mathrm{d}} \mathbb{E}_{S}\left[\hat{S}_{\mathrm{t}}-\mathrm{S}\left(\mathrm{x}_{\mathrm{t}}\right)\right]^{2} \leq \mathrm{C}_{6}\left(\varepsilon^{2}+\phi_{\varepsilon}^{2}+\frac{\varepsilon^{2}}{\phi_{\varepsilon}} \phi_{\varepsilon}^{2 \mathrm{HK}-1}\right) .
$$

Finally, under the assumption (H5), we obtain

$$
\lim _{\varepsilon \longrightarrow 0} \sup _{S(x) \in \Sigma_{0}(L)} \sup _{c \leq t \leq d} \mathbb{E}_{S}\left[\hat{S}_{t}-S\left(x_{t}\right)\right]^{2}=0 .
$$

\subsection{Proof of Theorem 2}

Using the Taylor formula, we get

$$
S\left(x_{t}\right)=S\left(x_{t_{0}}\right)+\sum_{j=1}^{k} S^{j}\left(x_{t_{0}}\right) \frac{\left(t-t_{0}\right)^{j}}{j !}
$$




$$
+\left(S^{k}\left(x_{t+\lambda\left(t-t_{0}\right)}\right)-S^{k}\left(x_{t_{0}}\right)\right) \frac{\left(t-t_{0}\right)^{k}}{k !}, \lambda \in(0,1)
$$

and

$$
\begin{aligned}
S\left(x_{\mathfrak{t}+\phi_{\varepsilon} \mathfrak{u}}\right)= & S\left(x_{\mathfrak{t}}\right)+\sum_{j=1}^{k} S^{j}\left(x_{\mathfrak{t}}\right) \frac{\left(\phi_{\varepsilon} u\right)^{j}}{j !} \\
& +\left(S^{k}\left(x_{t+\lambda\left(\phi_{\varepsilon} \mathfrak{u}\right)}\right)-S^{k}\left(x_{\mathfrak{t}}\right)\right) \frac{\left(\phi_{\varepsilon} u\right)^{k}}{k !}, \lambda \in(0,1) .
\end{aligned}
$$

Then, by substituting this expression in $\mathrm{I}_{2}$, using inequality (7) and assumptions (H6)-(H7), we obtain

$$
\begin{aligned}
I_{2} & =3 \mathbb{E}_{S}\left[\frac{1}{\phi_{\varepsilon}} \int_{0}^{T} G\left(\frac{\tau-t}{\phi_{\varepsilon}}\right) S\left(x_{\tau}\right) d \tau-S\left(x_{t}\right)\right]^{2} \\
& =3 \mathbb{E}_{S}\left[\int_{-\infty}^{+\infty} G(u) S\left(x_{t+\phi_{\varepsilon} u}\right) d u-S\left(x_{t}\right)\right]^{2} \\
& =3 \mathbb{E}_{S}\left[\int_{-\infty}^{+\infty} G(u)\left(S\left(x_{t+\phi_{\varepsilon} u}\right)-S\left(x_{t}\right)\right) d u\right]^{2} \\
& =3 \mathbb{E}_{S}\left[\int_{-\infty}^{+\infty} G(u)\left(\sum_{j=1}^{k} S^{j}\left(x_{t}\right) \frac{\left(\phi_{\varepsilon} u\right)^{j}}{j !}+\left(S^{k}\left(x_{t+\lambda\left(\phi_{\varepsilon} u\right)}\right)-S^{k}\left(x_{t}\right)\right) \frac{\left(\phi_{\varepsilon} u\right)^{k}}{k !}\right) d u\right]^{2} \\
& =3 \mathbb{E}_{S}\left[\frac{\phi_{\varepsilon}^{k}}{k !} \int_{-\infty}^{+\infty} G(u) u^{k}\left(S^{k}\left(x_{t+\lambda\left(\phi_{\varepsilon} u\right)}\right)-S^{k}\left(x_{t}\right)\right) d u\right]^{2}(b y u \operatorname{sing}(H 6)) \\
& \leq 3 C_{7}^{2} L^{2}\left[\frac{\phi_{\varepsilon}^{k+1}}{k !} \int_{-\infty}^{+\infty} G(u) u^{k+1} d u\right]^{2} \\
& \leq 3 C_{7}^{2} L^{2}(B-A) \frac{\phi_{\varepsilon}^{2(k+1)}}{(k !)^{2}}\left[\int_{-\infty}^{+\infty} G^{2}(u) u^{2(k+1)} d u\right] \\
& \leq C_{8} \phi_{\varepsilon}^{2(k+1)},
\end{aligned}
$$

where $C_{8}$ is a positive constant depending on $L$ and $(B-A)$. Next, from (14), (16), and (17), we find

$$
\sup _{S(x) \in \Sigma_{k}(L)} \sup _{c \leq t \leq d} \mathbb{E}_{S}\left|\hat{S}_{t}-S\left(x_{t}\right)\right|^{2} \leq C_{9}\left(\varepsilon^{2} \phi_{\varepsilon}^{2 H K-2}+\phi_{\varepsilon}^{2(k+1)}+\varepsilon^{2}\right) .
$$


Putting $\phi_{\varepsilon}=\varepsilon^{\frac{1}{\mathrm{k}-\mathrm{HK}+2}}$, it yields

$$
\limsup _{\varepsilon \longrightarrow 0} \sup _{S(x) \in \Sigma_{k}(L)} \sup _{c \leq t \leq d} \mathbb{E}_{S}\left(\left|\hat{S}_{t}-S\left(x_{t}\right)\right|^{2}\right) \varepsilon^{\frac{-2(k+1)}{k-H K+2}}<\infty .
$$

This completes the proof of Theorem 2 .

\subsection{Proof of Theorem 3}

From (4) and (10), we can see that

$$
\begin{aligned}
\varepsilon^{\frac{-(k+1)}{k-H K+2}}\left(\hat{S}_{t}-S\left(x_{t}\right)\right)=\varepsilon^{\frac{-(k+1)}{k-H K+2}}\left[\frac{1}{\phi_{\varepsilon}} \int_{0}^{T} G\left(\frac{\tau-t}{\phi_{\varepsilon}}\right)\left(S\left(X_{\tau}\right)-S\left(x_{\tau}\right)\right) d \tau\right. \\
\left.\quad+\frac{1}{\phi_{\varepsilon}} \int_{0}^{T} G\left(\frac{\tau-t}{\phi_{\varepsilon}}\right) S\left(x_{\tau}\right) d \tau-S\left(x_{t}\right)+\frac{\varepsilon}{\phi_{\varepsilon}} \int_{0}^{T} G\left(\frac{\tau-t}{\phi_{\varepsilon}}\right) d B_{\tau}^{H, K}\right] .
\end{aligned}
$$

Therefore

$$
\begin{array}{r}
\varepsilon^{\frac{-(k+1)}{k-H K+2}}\left(\hat{S}_{t}-S\left(x_{t}\right)\right)=\varepsilon^{\frac{-(k+1)}{k-H K+2}}\left[\int_{-\infty}^{+\infty} G(u)\left(S\left(X_{t+\phi_{\varepsilon}} u\right)-S\left(x_{t+\phi_{\varepsilon} u}\right)\right) d u\right. \\
\left.+\int_{-\infty}^{+\infty} G(u)\left(S\left(x_{t+\phi_{\varepsilon} u}\right)-S\left(x_{\mathfrak{t}}\right)\right) d u+\frac{\varepsilon}{\phi_{\varepsilon}} \int_{0}^{T} G\left(\frac{\tau-t}{\phi_{\varepsilon}}\right) d S_{\tau}^{H}\right] .
\end{array}
$$

Thus

$$
\varepsilon^{\frac{-(k+1)}{k-H K+2}}\left(\hat{S}_{t}-S\left(x_{t}\right)\right)=r_{1}(t)+r_{2}(t)+\eta_{\varepsilon}(t) .
$$

Hence, by Slutsky's Theorem, it suffices to show the following three claims:

$$
\begin{aligned}
& r_{1}(t) \rightarrow 0 \text {, as } \varepsilon \rightarrow 0 \text { in probability. } \\
& r_{2}(t) \rightarrow m \text {, as } \varepsilon \rightarrow 0 \text { in probability. }
\end{aligned}
$$

and

$$
\eta_{\varepsilon}(t) \rightarrow \mathcal{N}\left(0, \sigma_{\mathrm{H}, \mathrm{K}}^{2}\right) \text {, as } \varepsilon \rightarrow 0 \text { in distribution. }
$$

Proof of (18).

Let

$$
r_{1}(t)=\varepsilon^{\frac{-(k+1)}{k-H K+2}} \int_{-\infty}^{+\infty} G(u)\left(S\left(X_{t+\phi_{\varepsilon} u}\right)-S\left(x_{t+\phi_{\varepsilon}} u\right)\right) d u .
$$

By applying the inequality (14), we have

$$
0 \leq \mathbb{E}\left[r_{1}^{2}(t)\right] \leq \varepsilon^{\frac{-2(k+1)}{k-H K+2}} I_{1} \leq C_{10} \varepsilon^{\frac{2(1-H K)}{k-H K+2}}
$$


Therefore, using the Bienaymé-Tchebychev's inequality, as $\varepsilon \longrightarrow 0$, we obtain, for all $\alpha>0$

$$
P\left(\left|r_{1}(t)\right|>\alpha\right) \leq \frac{\mathbb{E}\left[r_{1}^{2}(t)\right]}{\alpha^{2}} \leq \frac{C_{10} \varepsilon^{\frac{2(1-H K)}{k-H K+2}}}{\alpha^{2}} \longrightarrow 0
$$

\section{Proof of (19).}

Let

$$
r_{2}(t)=\varepsilon^{\frac{-(k+1)}{k-H K+2}} \int_{-\infty}^{+\infty} G(u)\left(S\left(x_{t+\phi_{\varepsilon} u}\right)-S\left(x_{t}\right)\right) d u .
$$

By taking any $t, u \in[0, T]$ and $b(x) \in \Sigma_{k+1}(L)$, via the Taylor expansion, we get

$$
\begin{aligned}
S\left(x_{t+\phi_{\varepsilon} u}\right)= & S\left(x_{t}\right)+\sum_{j=1}^{k} S^{j}\left(x_{\mathfrak{t}}\right) \frac{\left(\phi_{\varepsilon} u\right)^{j}}{j !}+\frac{S^{k+1}\left(x_{\mathfrak{t}}\right)}{(k+1) !}\left(\phi_{\varepsilon} u\right)^{k+1} \\
& +\left(S^{k+1}\left(x_{t+\lambda\left(\phi_{\varepsilon} u\right)}\right)-S^{k+1}\left(x_{t}\right)\right) \frac{\left(\phi_{\varepsilon} u\right)^{k+1}}{(k+1) !}, \lambda \in(0,1),
\end{aligned}
$$

Making use of the conditions $(\mathrm{H} 6),(\mathrm{H} 7)$, and choosing $\phi_{\varepsilon}=\varepsilon^{\frac{1}{\mathrm{k}-\mathrm{HK}+2}}$, we obtain

$$
\begin{aligned}
\mathbb{E}\left[r_{2}(t)-m\right]^{2} & =\mathbb{E}\left[\int_{-\infty}^{+\infty} G(u)\left(S^{k+1}\left(x_{t+\lambda\left(\phi_{\varepsilon} u\right)}\right)-S^{k+1}\left(x_{t}\right)\right) \frac{(u)^{k+1}}{(k+1) !} d u\right]^{2} \\
& \leq C_{11} L^{2} C^{2}\left(\int_{-\infty}^{+\infty} G(u) u^{k+2} \frac{\phi_{\varepsilon}}{(k+1) !} d u\right)^{2} \\
& \leq C_{12}\left(\int_{-\infty}^{+\infty} G^{2}(u) u^{2(k+2)} d u\right) \phi_{\varepsilon}^{2} \\
& \leq C_{13} \phi_{\varepsilon}^{2}
\end{aligned}
$$

where $C_{13}$ is a positive constant which depends on $L$ and $k$, and

$$
m=\frac{S^{k+1}\left(x_{t}\right)}{(k+1) !} \int_{-\infty}^{+\infty} G(u) u^{k+1} d u .
$$

Therefore,

$$
\mathbb{E}\left[\mathrm{r}_{2}(\mathrm{t})-\mathrm{m}\right]^{2} \longrightarrow 0 \text { as } \varepsilon \longrightarrow 0
$$

Then

$$
r_{2}(t) \stackrel{\mathbb{P}}{\longrightarrow} \mathrm{m} .
$$

\section{Proof of (20).}

Let

$$
\eta_{\varepsilon}(t)=\varepsilon^{\frac{-(k+1)}{k-H K+2}} \varepsilon \phi_{\varepsilon}^{-1} \int_{0}^{T} G\left(\frac{\tau-t}{\phi_{\varepsilon}}\right) d B_{\tau}^{H, K} .
$$


In fact, we have to evaluate the variance of (21). To this end, let

$$
\mathbb{E}\left[\eta_{\varepsilon}(t)\right]^{2}=\left(\varepsilon^{\frac{1-H K}{k-H K+2}} \phi_{\varepsilon}^{-1}\right)^{2} \mathbb{E}\left(\int_{0}^{T} G\left(\frac{\tau-t}{\phi_{\varepsilon}}\right) d B_{\tau}^{H, K}\right)^{2} .
$$

Moreover, using equation (2), we have

$$
\mathbb{E}\left[\eta_{\varepsilon}(t)\right]^{2}=\left(\varepsilon^{\frac{1-H K}{k-H K+2}} \phi_{\varepsilon}^{-1}\right)^{2}\left[\phi_{\varepsilon}^{2 H K} \int_{-\infty}^{+\infty} \int_{-\infty}^{+\infty} G(u) G(v) \frac{\partial^{2} R_{H, K}(u, v)}{\partial u \partial v} d u d v\right] .
$$

Then, by taking $\phi_{\varepsilon}=\varepsilon^{\frac{1}{k-H K+2}}$, we get

$$
\mathbb{E}\left[\eta_{\mathcal{\varepsilon}}(\mathrm{t})\right]^{2}=\int_{-\infty}^{+\infty} \int_{-\infty}^{+\infty} \mathrm{G}(\mathrm{u}) \mathrm{G}(v) \frac{\partial^{2} R_{\mathrm{H}, \mathrm{K}}(\mathrm{u}, v)}{\partial u \partial v} \mathrm{dud} v
$$

with

$$
\frac{\partial^{2} R_{H, K}(u, v)}{\partial u \partial v}=\alpha_{H, K}\left(u^{2 H}+v^{2 H}\right)^{K-2}(u v)^{2 H-1}+\beta_{H, K}|u-v|^{2 H K-2},
$$

where

$$
\alpha_{\mathrm{H}, \mathrm{K}}=2^{-\mathrm{K}+2} \mathrm{H}^{2} \mathrm{~K}(\mathrm{~K}-1) \quad \text { and } \quad \beta_{\mathrm{H}, \mathrm{K}}=2^{-\mathrm{K}+1} \mathrm{HK}(2 \mathrm{HK}-1) .
$$

Finally, this last equation allows us to achieve the proof of Theorem 3.

\section{Conclusion}

This paper considered a nonparametric estimation of trend function for stochastic differential equations driven by a bifractional Brownian motion. We constructed an estimate of the trend function. Then, under some assumptions, we established the uniform consistency, the rate of convergence and the asymptotic normality of the proposed estimator. Further, an numerical example is provided. The present study has many applications in practical phenomena including telecommunications and economics.

\section{Acknowledgements}

The authors are thankful to the Editor-in-Chief and anonymous reviewers for their comments and valuable suggestions in improving the quality of this paper. 


\section{References}

[1] X. Bardina, K. Es-Sebaiy, An extension of bifractional Brownian motion, Communications on Stochastic Analysis, 5 (2) (2011), 333-340.

[2] K. Es-Sebaiy and C. A. Tudor, Multidimensional bifractional Brownian motion: Itô and Tanaka formulas, Stoch. Dyn., 7 (3) (2007), 365-388.

[3] C. Houdré and J. Villa, An example of infinite dimensional quasi-helix, Stochastic models (Mexico City, 2002), Contemp. Math., 336 (2003), 195201.

[4] J. P. Kahane, Hélices et quasi-hélices, Adv. Math., 7B (1981), 417-433.

[5] A. N. Kolmogorov, Wienersche Spiralen und einige andere interessante Kurven im Hilbertschen Raum. C.R. (Doklady) Acad. USSR (N.S.), 26 (1940), 115-118.

[6] I. Kruk, F. Russo, and C. A. Tudor, Wiener integrals, Malliavin calculus and covariance measure structure, J. Funct. Anal., 249 (1) (2007), 92142.

[7] Y. A. Kutoyants, Identification of dynamical systems with small noise, Springer Science \& Business Media, 300 (2012).

[8] M. Lifshits, K. Volkova, Bifractional Brownian motion: Existence and Border cases, preprint. http://arxiv.org/pdf/1502.02217.pdf., (2015).

[9] B. B. Mandelbrot, J. W. Van Ness, Fractional Brownian motions, fractional noises and applications, SIAM Rev., 10 (1968), 422-437.

[10] M. N. Mishra and B. L.S. Prakasa Rao, Nonparameteric Estimation of Trend for Stochastic Differential Equations Driven by Fractional Brownian Motion, Stat. Inference. Stoch. Process., 14 (2011), 101-109.

[11] M. N. Mishra, B. L. S. Prakasa Rao, Nonparametric Estimation of Linear Multiplier for Fractional Diffusion processes, Stochastic Analysis and Application, 29 (2011), 706-712.

[12] I. Norros, E. Valkeila, J. Virtamo, An elementary approach to a Girsanov formula and other analytical results on fractional Brownian motions, Bernoulli, 5 (4) (1999), 571-587. 
[13] B. L. S. Prakasa Rao, Nonparametric estimation of trend for stochastic differential equations driven by mixed fractional Brownian motion, Stochastic Analysis and Applications, 37 (2) (2019), 271-280.

[14] F. Russo and C. Tudor, On the bifractional Brownian motion, Stoch. Process. Their Appl., 116 (5) (2006), 830-856.

[15] B. Saussereau, Nonparametric inference for fractional diffusion, Bernoulli, 20 (2) (2014), 878-918.

[16] C. A. Tudor, Y. Xiao, Sample path properties of bifractional Brownian motion, Bernoulli 13 (2007), 1023-1052.

[17] L. Yan, J. Liu, and G. Jing, Quadratic covariation and Itô formula for a bifractional Brownian motion, preprint (2008).

Received: June 15, 2019 